\title{
COERCIÓN ASPECTUAL Y CONSTRUCCIÓN PROGRESIVA: EL ENFOQUE DE LA GRAMÁTICA DE CONSTRUCCIONES*
}

\author{
Nicolás Saavedra Garretón**
}

\section{Resumen}

En este artículo presentamos, en términos muy generales, los supuestos básicos del enfoque teórico conocido como Gramática de Construcciones (GC), para luego exponer en detalle el modo en que, desde esta perspectiva, se analiza el fenómeno semántico-gramatical de la coerción aspectual, con referencia a ejemplos del español. Para llevar a cabo esta exposición, nos concentramos en la propuesta de Michaelis (2004), que destaca tanto por su cuidadoso desarrollo como por su rigor formal.

Palabras clave: gramática de construcciones, coerción aspectual, construcción progresiva, semántica, gramática.

\section{Aspectual COERCIÓN AND THE PROGRESSIVE CONSTRUCTION: THE POINT OF VIEW OF CONSTRUCTION GRAMMAR}

\begin{abstract}
In this article we present, in very general terms, the basic assumptions of the theoretical approach known as Construction Grammar (CG), and then we develop a detailed account of the analysis of the semantic and grammatical phenomenon known as aspectual coercion, from the standpoint of $C G$, and with reference to Spanish examples. We base this exposition in the proposal made in Michaelis (2004), because it stands out in terms of its thorough development and formal rigour.
\end{abstract}

Keywords: Construction Grammar, aspectual coercion, progressive construction, semantics, grammar.

* Este artículo se inscribe en la Tesis Doctoral apoyada por la Beca Conicyt N ${ }^{\circ}$ D-21060282. Título de la tesis: "Partes de Eventos: Coerción aspectual en el sintagma verbal español"

** Chileno. Doctor en Lingüística de la Universidad de Concepción. Académico en la Facultad de Letras de la Pontificia Universidad Católica de Chile, Santiago, Chile. lsaavedrag@uc.cl 


\section{Introducción: la coerción aspectual en Gramática de Construcciones}

La Gramática de Construcciones (en adelante, GC) es una teoría gramatical que ubica en el centro de los fenómenos lingüísticos la noción de construcción. Las construcciones corresponden a "stored pairings of form and function, including morphemes, words, idioms, partially lexically filled and fully general linguistic patterns." (Goldberg, 2007, p. 590). Una definición más general es la de Kay y Fillmore (1999): “A construction (...) is a set of conditions licensing a class of actual constructs of a language (...)" (p.2). Estas dos definiciones pueden articularse del siguiente modo: los binomios a los que alude Goldberg corresponden a conjuntos de condiciones y requerimientos que definen, colectivamente, criterios de "buena formación" (e.g. gramaticalidad) para los distintos estratos morfo-sintácticos de una lengua natural. Las subsecciones siguientes ejemplificación la aplicación de esta concepción lingüística al estudio del fenómeno de la coerción aspectual.

\section{El tratamiento de la coerción aspectual en GC: El modelo de dos niveles de Michaelis (2004)}

El trabajo de Michaelis (2004) sobre coerción aspectual ${ }^{1}$ asume un modelo de representación de clases léxicas aspectuales (e.g. clases de Aktionsart) de dos niveles. La justificación de esta elección se halla en lo que la autora considera como un doble carácter de la estructura de eventos: en un nivel, ésta se halla configurada como un evento causativo idealizado, y en otro, como una estructura continua que ocupa intervalos temporales de tamaño variable (p.12). A continuación ofrecemos una caracterización sucinta de ambos niveles.

1 En tanto fenómeno interpretativo, la coerción aspectual corresponde a la transición desde una estructura eventiva asociada por defecto a un predicado, hacia una estructura distinta que provee una interpretación también diferente. A modo de ilustración, cabe señalar la transición de actividad a efectuación en, respectivamente, María dibujó círculos y María dibujó un círculo. Aquí, 'actividad' y 'efectuación' corresponden, por supuesto, a dos tipos de evento pertenecientes a la tipología clásica de Vendler (1967). Una definición más extensa de la noción de coerción aspectual puede encontrarse en la sección 1 de Michaelis (2004). 


\subsection{Representación causal (RC)}

El modelo de RC que presenta Michaelis (2004) está basado en el trabajo de Rappaport Hovav y Levin (1998). Su contenido central está expuesto en (1) (p.13):

(1) Representaciones causales de las clases de Aktionsart:

\begin{tabular}{|c|c|}
\hline Clase de Aktionsart & $\begin{array}{l}\text { Representación causal (Plantillas de estructura } \\
\text { de evento o PEEs) }\end{array}$ \\
\hline Estado & {$[x<$ ESTADO $>$ ] e.g. creer en Dios, saber inglés } \\
\hline Fase de estado & $\begin{array}{l}\text { [MANTENER }[x<\text { ESTADO }>\text { ]] e.g. estar } \\
\text { enfermo por dos días }\end{array}$ \\
\hline $\begin{array}{l}\text { Actividad } \\
\text { homogénea }\end{array}$ & [x MANTENER $[x<$ ESTADO $>$ ]] e.g. dormir \\
\hline $\begin{array}{l}\text { Actividad } \\
\text { heterogénea }\end{array}$ & [x REPETIR $[x<$ EVENTO $>$ ]] e.g. correr \\
\hline Logro & $\begin{array}{l}{[\text { DEVENIR }[x<\text { ESTADO }>\text { ]] e.g. hundirse, }} \\
\text { empezar a trabajar, terminar la reunión, } \\
\text { descubrir la fórmula }\end{array}$ \\
\hline Realización & $\begin{array}{l}{[[[x \text { REPETIR }[x<\text { EVENTO }>]] \text { CAUSA }} \\
[\text { DEVENIR }[y<\text { ESTADO }>]]] \text { e.g. construir una } \\
\text { casa, hacer una torta }\end{array}$ \\
\hline
\end{tabular}

En el modelo original de Rappaport Hovav y Levin (1998), cada una de las plantillas de estructura de evento que acompañan a cada clase de aktionsart, equivale a una combinación de componentes capturados a partir de lo que en Rappaport Hovav y Levin (1998) se denomina "descomposición de predicados" (p.104). Las descomposiciones de predicados son asignadas a los miembros de las distintas clases aspectuales y, por ende, permiten caracterizarlos. En general, estas descomposiciones de predicados corresponden a estructuras eventivas complejas. Los componentes obtenidos a partir de esta descomposición equivalen a predicados primitivos (correspondientes en (1) a los operadores en mayúsculas reducidas), "constantes" (correspondientes en (1) a los segmentos anotados entre paréntesis angulados) y variables individuales. Michaelis incorpora estas formulaciones a su propuesta, agregando tres nuevos tipos aspectuales: el tipo 'Fases de estado', más dos sub-tipos del tipo tradicional de actividad: 'Actividad homogénea' y 'Actividad heterogénea'. 
1.1.1.1.1 El proceso recursivo de combinación de PEEs para formar otras PEEs es denominado, por Rappaport Hovav y Levin (1998) (y, a partir de esta formulación, por Michaelis (2004)) aumento de plantilla (AP). El AP posee dos restricciones:

\section{2}

(2) a. Restricción de mapeo por pares: el AP sólo permite mapeos de un tipo aspectual de entrada a un tipo aspectual de salida (representados ambos por PEEs).

b. Restricción de sub-operador: sólo un operador sub-eventivo puede agregarse a los tipos de entrada (Michaelis, 2004, p. 14).

En el ejemplo (3), el tipo aspectual 'Realización' denota una función que mapea a los tipos aspectuales 'Actividad heterogénea' y 'Logro' (por separado) a 'Realización' con la adición posible de un solo operador subeventivo a cada aducto y en cada caso. Así:

(3) Función denotada por el tipo aspectual 'Realización'

$<$ [x REPETIR $[x<$ EVENTO $>]]$,

[[[x REPETIR [ $x<$ EVENTO>]] CAUSA [DEVENIR [y $<$ ESTADO>]]]> Aducto (tipo aspectual 'Actividad heterogénea'):

$x[x$ REPETIR $[x<$ EVENTO $>]]$

Educto:

$=[[[x$ REPETIR $[\mathrm{x}<$ EVENTO $>]]$ CAUSA [DEVENIR $[y<$ ESTADO $>]]]$

(4) Función denotada por el tipo aspectual 'Realización':

$<$ CAUSA [DEVENIR [y $<$ ESTADO $>$ ]],

[[[x REPETIR [ $x<$ EVENTO>]] CAUSA [DEVENIR [y $<$ ESTADO> $]]]>$ Aducto de tipo aspectual 'logro' con operador sub-eventual agregado: $\times$ CAUSA [DEVENIR $[\mathrm{y}<$ ESTADO $>$ ]]

Educto:

$=[[[x$ REPETIR $[\mathrm{x}<$ EVENTO $>]]$ CAUSA [DEVENIR $[y<$ ESTADO $>]]]$ 
Como puede observarse, en el caso de (4), el tipo de aducto, correspondiente a 'Logro', lleva consigo un operador sub-eventivo (CAUSA). Según la restricción de (2b), este tipo de entrada ya no puede recibir la adición de un nuevo operador.

\subsection{Representación temporal (RT)}

Para Michaelis (2004), toda situación (en tanto supercategoría que engloba tanto a los eventos como a los estados) puede caracterizarse a partir de patrones de estado y cambio que la autora representa haciendo uso de tipos temporales básicos: estados $(\varphi)$, transiciones $(\tau)$, y cadenas de eventos (к). Este modelo está basado en Bickel (1997), para quien la ' $\varphi$ ' simboliza fases y la ' $\tau$ ' representa transiciones o límites (boundaries) (p. 116). El símbolo para las cadenas de eventos es una contribución de Michaelis, quien propone las siguientes representaciones temporales para los distintos tipos de Aktionsart:

(6) Representaciones temporales en Michaelis (2004, p.15):

\begin{tabular}{ll}
\hline Clase de Aktionsart & Representación temporal (RT) \\
\hline Estado & $\varphi$ \\
\hline Fase de estado & $\tau \varphi \tau$ \\
\hline Actividad homogénea & $\tau \varphi \tau$ \\
\hline Actividad heterogénea & $\tau \varphi[\tau \varphi]+\tau$ \\
\hline Logro & $\tau \varphi$ \\
\hline Efectuación & $\kappa \tau \varphi$ \\
\hline
\end{tabular}

Una limitación fácilmente observable de la RT, consiste en que no permite distinguir notacionalmente la clase 'Fase de estado' de la clase 'Actividad heterogénea'. Para efectuar dicha distinción se hace imprescindible la complementación de la RT con la RC.

\section{Operaciones de cambio de tipo aspectual en Michaelis}

En la propuesta de Michalis (2004), los mapeos relevantes están restringidos por un principio general que la autora denomina 'Principio de preservación de aktionsart', descrito a continuación: 
(7) Principio de preservación de Aktionsart (PPA): "In an aspectual mapping, whether implicit or explicit, input and output types must share some portion of their respective causal and/or temporal representations" (Michaelis, 2004, p. 16).

Este principio limita las operaciones posibles sobre la estructura de Aktionsart que generan cambios de tipo aspectual. Consideremos, al respecto, el procedimiento de permutación, que se define a continuación:

(8) Permutación: "Permutation operations add or select a single component of the input Aktionsart representation" (Michaelis, 2004, p. 16).

Considerando que en este modelo se aceptan dos representaciones complementarias de estructura eventiva (RC y RT), la permutación puede agregar o seleccionar el componente del caso en cualquiera de éstas. En particular, un componente de RC es un operador y los argumentos que proyecta, y uno de RT es un estado, una transición o una cadena de eventos (p.17). Michaelis ofrece el siguiente ejemplo de adición, en una transición de estado a realización:

(9) They were bored in a few seconds.

La autora propone que, en este ejemplo, la combinación del adverbio de marco temporal 'in a few seconds', con un predicado de estado ('they were bored'), gatilla la adición del operador DEVENIR a la RC del estado o, equivalentemente, la adición de una transición a la RT del mismo.

Los ejemplos de selección que ofrece (p. 17) corresponden a instancias de la construcción copulativa resultativa del inglés:

(10) a. The truck is loaded.

b. The soup is cooled.

La autora señala que los estados resultativos denotados por ambos ejemplos ('being loaded' y 'being cooled', respectivamente) son el educto de una función que mapea un logro a un estado, en el caso de (10a), y de una función que mapea una realización a un estado, en el caso de (10b) (Michaelis, 2004, p. 17).

Sobre la base de este modelo, las operaciones de permutación cumplen un rol central en el cambio de tipo aspectual implícito presente en la construcción progresiva, como veremos más adelante. Adicionalmente, 
las operaciones que participan en dicha construcción parecen violar el PPA, como señala Michaelis (2004). Sin embargo, esta violación sólo puede afirmarse cuando se acepta que los únicos mapeos posibles consisten en un par ordenado cuyo primer elemento es un tipo aspectual de aducto básico (en RC o RT) y cuyo segundo elemento es un tipo aspectual de educto (en RC o RT) análogo. Una consideración así es posible sólo si se ignora el hecho de que las funciones no están restringidas a mapeos entre tipos básicos: las funciones de funciones a funciones (o, alternativamente, los mapeos entre funciones) no representan un problema, pues no es teóricamente inconsistente que tanto la entrada como la salida de un mapeo, o función, correspondan ambas a pares ordenados.

\section{El Principio de Cancelación en Michaelis}

Michaelis (2004) propone un mecanismo de acomodación del significado léxico que provee una explicación general para el fenómeno de coerción aspectual. Este mecanismo es llamado por la autora principio de cancelación (Override principle), y es definido como sigue:

(11) Principio de Cancelación: "If a lexical item is semantically incompatible with its morphosyntactic context, the meaning of the lexical item conforms to the meaning of the structure in which it is embedded" (p. 25).

La aplicación de este principio es ejemplificada por Michaelis en un caso de coerción nominal observable en ciertas combinaciones de unidades léxicas con la construcción de plural. En los ejemplos de (12) la expresión (a) corresponde a un caso de ajuste léxico y la expresión (b) a uno de desajuste léxico, que se resuelve por coerción:

(12) a. She bought some pencils.

b. They serve delicious soups

(p. 26).

(13) La construcción de plural (adaptada de Michaelis, 2004, p. 27): 

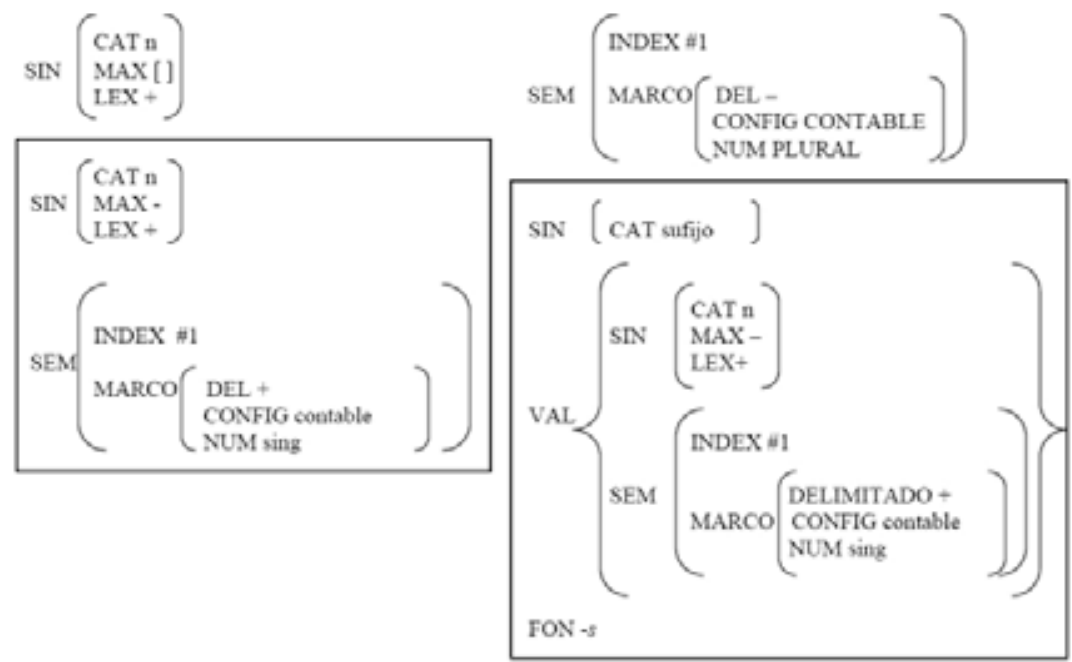

En (13) puede observarse que la construcción de plural es una función que mapea una unidad léxica nominal de entrada a una unidad léxica nominal de salida con rasgos semánticos distintos a la unidad de entrada. La construcción posee un requerimiento de valencia (rasgo VAL) en el nodo hijo de la derecha (el sufijo de plural) con el cual debe unificarse el nodo de la izquierda, que recibe la entrada léxica (la raíz nominal). No obstante lo anterior, señala Michaelis, no existe ningún caso en que la entrada léxica satisfaga el valor atómico ('-') establecido para el rasgo DEL (Delimitación) del nodo madre (p. 28): "The plural construction shifts the boundedness value of the input noun to [bounded -], producing forms like soups" (p. 28). En otras palabras, la construcción de plural efectúa por defecto un cambio de tipo sobre el valor para DEL del nodo hijo de la derecha. Se trata de una función específica de esta construcción: transformar el valor DEL + del nodo hijo de la derecha a un valor DEL-.

Cuando la unidad léxica de entrada satisface este rasgo del nodo hijo nominal de la construcción, se produce lo que Michaelis denomina 'ajuste léxico' (lexical match) (p. 26), como en (12a). En este caso, la unidad léxica de entrada ('pencil') satisface el rasgo DEL+ requerido por el nodo hijo nominal. Este rasgo será cambiado de todas formas a DEL- por el nodo madre, una vez que la unidad léxica de entrada haya satisfecho los requerimientos VAL de su nodo hermano y se haya unificado con este mismo nodo. En cambio, cuando la unidad léxica de aducto es DEL- (e.g. soup), ésta fracasa en satisfacer los requerimientos del nodo nominal de 
la construcción: se trata de un caso de desajuste léxico (lexical mismatch) (p. 26). El principio de cancelación predice que el conflicto entre unidad de entrada y requerimiento construccional se resolverá en favor de la construcción: el rasgo DEL- de la unidad léxica será cambiado a DEL+ por la construcción, para luego ser cambiado nuevamente a DEL- cuando la unidad logre unificarse con los requerimientos VAL del nodo hijo de la derecha. Michaelis (2004) plantea que, en este caso, nos encontramos antes dos transiciones de tipo distintas. La primera modifica un rasgo de la unidad léxica de entrada para resolver un conflicto entre ésta unidad y la construcción, según el principio de cancelación. La segunda modifica un rasgo requerido por uno de los nodos hijo de la construcción (consiguientemente, se trata de un requerimiento construccional). Este segundo cambio de tipo no es, por tanto, producto de un conflicto semántico, sino que responde más bien al "diseño" específico de la construcción y en él no opera el principio de cancelación. El primer tipo de transición representa lo que Michaelis denomina transición implícita de tipo (implicit type shifting) (p. 28) que caracteriza, propiamente, lo que hemos denominado hasta aquí "coerción". El segundo tipo de transición representa lo que Michaelis denomina transición explícita de tipo (explicit type shifting) (Michaelis, 2004) y describe un fenómeno distinto a la coerción. La coerción, por tanto, presupone la aplicación del principio de cancelación y es equivalente a la transición de tipo implícita. Como veremos en la sección siguiente, la transición de tipo implícita, entendida en los términos aquí expuestos, permite explicar, en una construcción verbal, fenómenos análogos a los recién ilustrados para el caso de la construcción de plural.

\section{El análisis de la construcción progresiva (perífrasis de gerundio con estar)}

La construcción progresiva es una construcción transicional estativizadora cuyo complemento SV posee, como valor para el rasgo marco, una representación temporal del tipo aspectual 'Actividad homogénea' (Michaelis, 2004, p. 35). En tanto, la representación temporal de la construcción corresponde al tipo aspectual 'Fase de estado' (p. 35). A continuación presentamos una representación de esta construcción:

(14) La construcción progresiva (perífrasis de gerundio con estar): 
$\sin \left(\begin{array}{c}\text { CAT v } \\ \text { MAX }+\end{array}\right)$

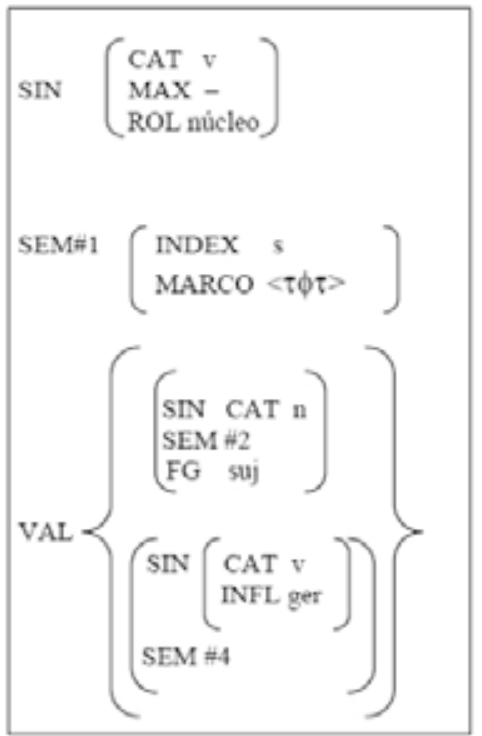

$\operatorname{SEM}\left(\begin{array}{l}\text { INDEX } s \\ \text { MARCO }\langle\tau \phi \tau, \tau \phi \tau\rangle \\ \text { ARG1 } \# 1 \\ \text { ARG2 } \# 5\end{array}\right)$

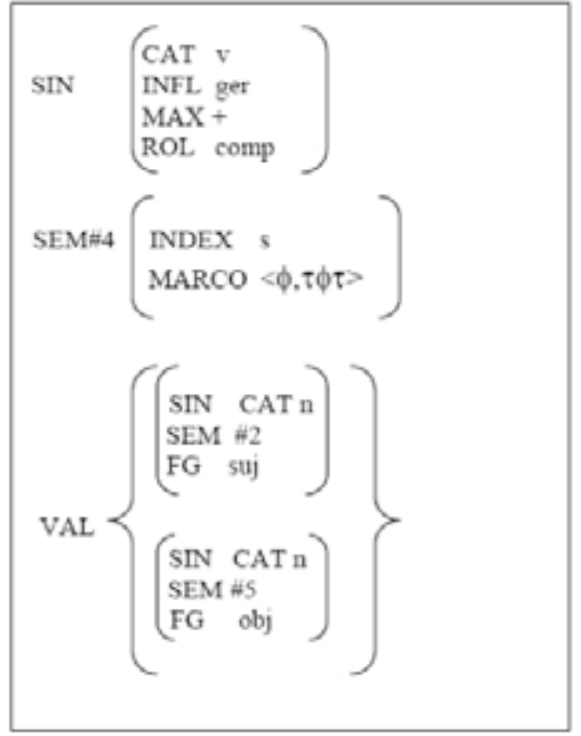

La construcción efectúa un cambio de tipo explícito: el tipo estativo que denota la construcción es el producto de una operación de selección sobre la actividad denotada por el complemento SV (sintagma verbal). Más detalladamente, la construcción selecciona un estado intermedio en la RT de la actividad (p. 35):

$<\tau \phi \tau, \tau \phi \tau>$

construcción: perífrasis de gerundio con estar; tipo aspectual: estado (durativo))

$\times \quad \tau \phi \tau$

(tipo aspectual del SV complemento: actividad homogénea)

$=\tau \phi \tau$

(selección: fase de estado) 
(15) Transición explícita de tipo aspectual (selección):

La coerción generada por la construcción progresiva se produce cuando la construcción recibe un tipo estativo de aducto en su SV complemento. Este tipo de aducto transgrede la exigencia construccional sobre el tipo aspectual del complemento, que debe corresponder, como ya vimos, a 'Actividad homogénea'. En cambio, cuando el tipo de aducto es estativo, la construcción realiza un cambio de tipo de 'Estado' a 'Actividad homogénea', mediante la operación de adición, y luego selecciona un estado intermedio de este último educto. Es decir, realiza una secuencia de cambios de tipo: primero uno implícito y luego otro explícito. La satisfacción de la exigencia construccional se explica según el principio de cancelación: la exigencia de la construcción (en este caso, de tipo aspectual) se impone sobre cualquier valor de un rasgo particular de la entrada léxica, si este último entra en conflicto con un valor para el mismo rasgo en la construcción. Podemos notar que el tipo 'Actividad homogénea' es requerido por los dos hijos de la construcción: el nodo de la izquierda (el núcleo de la construcción) requiere un complemento de tipo 'Actividad homogénea' en el rasgo VAL. Esto queda establecido por el valor de unificación \#4 en SEM. En tanto, el nodo hijo de la derecha (el complemento de la construcción), requiere este tipo aspectual como un valor intrínseco para el rasgo MARCO de SEM.

La construcción progresiva ha servido de prueba para identificar verbos léxicamente estativos. Estos últimos, se ha afirmado, pueden caracterizarse, al menos parcialmente, a partir de su incompatibilidad con esta construcción (De Miguel, 1999, p. 3013; Morimoto, 1998, p. 20). Ejemplos relevantes de esta incompatibilidad que pueden encontrarse en la literatura sobre aspecto léxico son los siguientes:

(16) a. *Juan está sabiendo la verdad.

b. *La familia está poseyendo la empresa.

c. *Juan está teniendo muchos libros.

d. *Juan está odiando a su primo.

A modo de explicación, se ha propuesto que tal incompatibilidad se basa en una anomalía lógica, pues resultaría "semánticamente contradictorio expresar el progreso en el tiempo de un evento que se caracteriza por no manifestar avance o cambio" (De Miguel, 1999, p. 
3013). Hay que señalar, además, que esta prueba de estatividad léxica ha sido propuesta en el contexto específico de la oposición estado/ actividad: la resistencia de verbos con aspecto léxico estativo a entrar en esta construcción contrasta con la compatibilidad de los verbos de actividad para participar en la misma:

(17) a. Juan está cantando.

b. Juan está corriendo.

c. Juan está estudiando.

d. Juan está bailando.

Para De Miguel (1999) la compatibilidad ejemplificada en (17) subrayaría la progresión de estos eventos dinámicos, que "implican un cambio o progreso a lo largo del intervalo de tiempo en que ocurren" ( $\mathrm{p}$. 3018). Aunque esta prueba parece ofrecer criterios claros de identificación de los tipos aspectuales 'Estado' y 'Actividad', tanto en el trabajo de De Miguel, como en el de Morimoto se indican casos excepcionales, en que los verbos estativos reciben una interpretación ingresiva cuando se combinan con la construcción (e.g. 'estoy conociendo a mucha gente', en Morimoto, 1998, p.20). De hecho, De Miguel (1998) señala que "casi todos los verbos estativos pueden entrar a formar parte de formas progresivas" (p. 3013). A este respecto, la autora ofrece los ejemplos de (18), que reproducimos a continuación:

(18) a. Te estoy queriendo cada vez más.

b. Juan está odiando a su primo en estos días más de lo que le habrán odiado en toda su vida.

c. Estoy sabiendo cada vez más cosas sobre ese amigo tuyo tan misterioso. Estos días estoy teniendo muchos problemas con el fax.

d. Últimamente estoy teniendo suerte en todo.

Para De Miguel(1999), la aparición de los verbos léxicamente estativos de (18) en la construcción progresiva responde a una determinación contextual, en la medida en que "el contexto ha contribuido a proporcionar una lectura dinámica, de acuerdo con la cual se describe un evento que avanza o progresa en fases sucesivas o una acumulación de estados que se repiten (...)" (p. 3014). Para la autora, los ejemplos de (18), resultan 
de contribuciones contextuales variadas: $(18 \mathrm{a}, \mathrm{b})$ son "predicados cuya intensidad da a entender que el evento avanza gradualmente: no son pues, homogéneos, sino dinámicos (p. 3014); en (18c) 'saber' recibe una lectura ingresiva: se trata del "comienzo de un estado o su desarrollo" (p. 3014); (18d) muestra una lectura dinámica gatillada por el núcleo del SN complemento directo (problemas), que "permite que el evento denote un hecho que está ocurriendo de manera repetida (...)" (p. 3014). Finalmente, en (18e) la lectura dinámica también estaría gatillada por el núcleo del SN complemento directo ('suerte'), aunque en este caso es el carácter denotativo de este nominal, de tipo acumulativo, el que produciría la lectura especial. Para De Miguel (1999), tanto en este ejemplo, como en el de (18d), el dinamismo de los predicados es de carácter específicamente iterativo: mientras 'últimamente estoy teniendo suerte' denota una repetición sucesiva del estado tener suerte en el intervalo denotado por 'últimamente', 'estos días estoy teniendo problemas' denota, de igual manera, el estado 'tener problemas' en el intervalo denotado por 'estos días'. En ambos casos, la semántica del núcleo del SN complemento directo (acumulativa en el caso de suerte; facilitadora de una lectura de repetición, en el caso de problemas) contribuyen a la lectura dinámica iterativa denotada por la construcción (p. 3014).

Aunque De Miguel no usa en estos desarrollos las nociones como 'coerción aspectual' o 'transición de tipo implícita', su explicación general de la lectura dinámica de verbos léxicamente estativos en los ejemplos de (18) alude a procesos interpretativos equivalentes:

(...) dado que la información aspectual de una oración no viene proporcionada en exclusiva por el aspecto léxico del $\mathrm{V}$, la presencia de determinados modificadores adverbiales o de ciertos CCDD puede dinamizar la información -estativaatribuida al verbo como unidad léxica; en este caso, el verbo queda capacitado para admitir la forma progresiva (...). (De Miguel, 1999, p. 3014)

En el caso de (18a-c), 'querer', 'odiar' y 'saber' reciben, en RT, la adición de transiciones que transforman a la representación de 'Estado' en una representación de 'Actividad homogénea'. Se trata de un proceso de coerción propiamente tal (y, como tal, presupone el principio construccional de cancelación), cuyo educto sirve, a su vez, para alimentar un nuevo proceso de transición aspectual, esta vez explícito, a 
saber: uno que transforma una 'Actividad homogénea' en el tipo estativo durativo denotado por la construcción completa. La operación completa puede representarse usando la notación de RT, en razón de su carácter abreviado en comparación con las representaciones de RC:

(19) Procesos de cambios de tipo aspectual para los ejemplos de (18a-c):

$<<<\phi, \tau \phi \tau>,<\tau \phi \tau, \tau \phi \tau>>\times<\phi, \tau \phi \tau>=<\tau \phi \tau, \tau \phi \tau>>\times \tau \phi \tau=\tau \phi \tau$ estar+gerundio: (coerción:adición); (transición explícita: selección en RC) e.g. queriendo, sabiendo; e.g. está queriendo, está sabiendo.

\section{Comentarios finales}

En este artículo hemos presentado la formulación explícita que ofrece Michaelis (2004) para el tratamiento de la coerción eventiva en GC, y vimos que dicha formulación corresponde, ampliamente, a un modelo basado en representaciones causales y temporales de la estructura de eventos denotada por los verbos y los sintagmas verbales. Al respecto, nos parece conveniente destacar la mayor riqueza descriptiva del sistema de representación causal (RC) que utiliza Michaelis, en comparación con el sistema de representación temporal (RT).

Adicionalmente a este sistema representacional basado en dos modos de conceptualización, Michaelis (2004) introduce un principio interpretativo que aplica globalmente a los casos de coerción: el 'principio de cancelación', que establece que si una unidad léxica es semánticamente incompatible con su contexto morfosintáctico, el significado de la unidad se ajusta al significado de la estructura en la cual participa. Claramente, este principio asume un fundamento básico de la GC, a saber, que las configuraciones morfosintácticas también poseen significado, pues, después de todo, el principio de cancelación atañe a una incompatibilidad semántica. La función que cumple este principio en el tratamiento de la coerción aspectual es clave, pues pretende explicar, precisamente, por qué se produce este fenómeno interpretativo.

Finalmente, hemos mostrado cómo las propuestas que Morimoto (1998) y De Miguel (1999) han ofrecido para explicar casos de coerción aspectual en la construcción progresiva del español son compatibles con el tratamiento que de este fenómeno se ha formulado en el marco de la Gramática de Construcciones. Se trata de una compatibilidad 
relevante pues puede servir de base para nuevas aplicaciones de mayor explicitud formal a la explicación y descripción de fenómenos semánticogramaticales del español.

\section{Bibliografía}

Bickel, B. (1997). Aspectual scope and the difference between logical and semantic representation. Lingua, 102, 115-131.

De Miguel, E. (1999). El aspecto léxico. En Bosque, I. y Violeta D (Eds.). Gramática descriptiva de la lengua española (pp. 29773060). Madrid: Espasa Calpe.

Goldberg, A. (2007). Constructions: A New Theoretical Approach to Language. En V. Evans, B. Bergen y J. Zinken. The Cognitive Linguistics Reader (pp. 589-600). Londres: Equinox

Kay, P. y Fillmore, C.J. (1999). Grammatical constructions and linguistic generalizations: the 'what's $\mathrm{X}$ doing $\mathrm{Y}^{\prime}$ construction, Language, 75, 1-33.

Michaelis, L. (2004). Type Shifting in Construction Grammar: An Integrated Approach to Aspectual Coercion. Cognitive Linguistics, 15, 1-67.

Michaelis, L. (2006). Construction Grammar. En Brown, K (Ed.). The Encyclopedia of Language and Linguistics (2da ed.). (Vol.3, pp.73-84). Oxford: Elsevier.

Morimoto, Y. (1998). El aspecto léxico: delimitación. Madrid: Arco Libros.

Rappaport Hovav, M. y Levin, B. (1998). Building verb meanings. En Butt,

M. y Geuder, W (Eds.). The Projection of Arguments (pp. 97-134). Stanford: CSLI Publications.

Vendler, Zeno. (1967). Linguistics in Philosophy. Londres: Cornell University Press. 